\title{
Association Between the Anti-Aging Protein Klotho and Sleep Duration in General Population
}

\author{
Dongdong Huang (D) \\ Saibin Wang (D) ${ }^{2}$ \\ 'Department of Respiratory and Critical \\ Care Medicine, The Fourth Affiliated \\ Hospital, Zhejiang University School of \\ Medicine, Yiwu, 322000, Zhejiang, \\ People's Republic of China; ${ }^{2}$ Department \\ of Respiratory Medicine, Affiliated Jinhua \\ Hospital, Zhejiang University School of \\ Medicine, Jinhua Municipal Central \\ Hospital, Jinhua, 321000, Zhejiang, \\ People's Republic of China
}

Correspondence: Saibin Wang Department of Respiratory Medicine, Affiliated Jinhua Hospital, Zhejiang University School of Medicine, Jinhua Municipal Central Hospital, Jinhua, 321000 , Zhejiang Province, People's Republic of China

Tel +8657982552278

Fax +8657982325006

Email saibinwang@hotmail.com
Purpose: Sleep duration is associated with aging. However, the relationship between sleep duration and the concentration of the protein klotho in the serum remains unknown in the general population of the United States. Hence, this study aimed at exploring the association between them.

Methods: Participants whose data included klotho protein and sleep duration variables in the National Health and Nutrition Examination Survey data from 2007 to 2016 were utilized for this analysis.

Results: Sleep duration was non-linearly associated with the level of klotho protein in the serum, with a negative association between sleep duration and serum klotho concentration after adjusting for confounding variables $(\beta=-7.6 ; 95 \% \mathrm{CI}:-11.3,-4.0 ; \mathrm{P}<0.001)$. The conversion of the sleep duration from a continuous variable to a categorical variable (tertile: T1: $<5.5$ hours; T2: 5.5-7.5 hours; T3: $>7.5$ hours) revealed that the serum klotho of the participants in the highest tertile ( $>7.5$ hours) was $21.9 \mathrm{pg} / \mathrm{mL}$ lower $(95 \% \mathrm{CI}:-38.6,-5.2$; $P=0.01)$ than those in the lowest tertile ( $<5.5$ hours).

Conclusion: Our results revealed that people who sleep more than 7.5 hours per night have decreased levels of the anti-aging protein klotho in their serum, thus being more at risk of aging-related syndromes.

Keywords: klotho protein, sleep duration, aging, NHANES

\section{Introduction}

The sleep duration recommended by the National Sleep Foundation in 2015 was as follows: 7-9 hours in young people and adults, and 7-8 hours in elderly people. Excessive or insufficient sleep duration is disadvantageous for health. Previous studies have shown that sleep duration is associated with cardiovascular disease, cognitive decline, and metabolic syndrome, ${ }^{1-3}$ and aging. ${ }^{4}$

Klotho protein is a multifunctional protein encoded by the klotho gene, and its expression level is associated with aging. ${ }^{5}$ Kuro-o found that mice lacking klotho suffer from premature aging syndrome, ${ }^{6}$ the lack of klotho in serum is also associated with heart aging, ${ }^{7,8}$ and decreased klotho levels are found in patients with various aging-related diseases, such as metabolic syndrome, cancer, and hypertension. ${ }^{9-11}$ In contrast, high level of klotho prolongs lifespan. ${ }^{6}$

Aging is an inevitable process for human beings. Although there is a fixed limit to human life span, ${ }^{12}$ the speed of aging is affected by many factors. Aging is affected by environmental, genetic, and epigenetic factors. ${ }^{13}$ On the other hand, the expression level of klotho may be potentially involved in the relationship between sleep duration and aging. Sleep disorders and aging are common public health 
problems, and the potential association between sleep duration and the anti-aging protein klotho is largely unexplored. Therefore, the purpose of this study was to investigate the potential association between them using the data of the National Health and Nutrition Examination Survey (NHANES) from 2007 to 2016, performed in the population of the United States. Our hypothesis is that sleep duration is associated with the serum anti-aging protein klotho concentration.

\section{Methods}

\section{Study Population}

The NHANES contains the data related to the anti-aging protein klotho for the following 5 cycles: 2007-2008, 2009-2010, 2011-2012, 2013-2014, 2015-2016. In this study, the National Center for Health Statistics was used to merge the publicly available documents of the 5 cycles of NHANES.

A total of 13,765 participants included in the NHANES database who had klotho in the serum measured from 2007 to 2016 were included in this study. Sleep indicators were considered for those participants who measured klotho.

\section{Klotho}

The serum klotho concentration in the participants was measured using a commercially available Enzyme Linked Immunosorbent Assay (ELISA) kit produced by ImmunoBiological Laboratories international in Japan. The serum samples of the participants were received on dry ice and stored at $-80^{\circ} \mathrm{C}$ until analysis. The samples were analyzed in duplicate, and the mean of the two values was used to calculate the final value. Two quality control samples containing low and high concentrations of klotho protein were also analyzed in duplicate by ELISA. Samples with more than $10 \%$ repeated results were considered as repeated analysis. If the values of the quality control samples were not within the 2SD range of the specified value, the entire analysis was discarded, and the sample analysis was repeated.

\section{Sleep-Related Variables}

The following self-reported outcomes related to sleep such as sleep duration and trouble sleeping were collected. These questions were asked at home by trained interviewers using the computer-assisted personal interview system.

Sleep duration: According to the questionnaire about the sleeping habits of the participants, the mean sleep duration per night was asked. The range of sleep duration was 1-12 hours, and the value of more than 12 hours was defined as 12 hours.

Trouble sleeping: Participants were asked whether they informed the doctor about their trouble sleeping. The answer to this question was divided into "Yes" or "No".

\section{Covariates}

Information about age (years), gender, race, education level, marital status, and income level was obtained from the demographic documents. Race was divided into Mexican American, other Hispanic, non-Hispanic white, non-Hispanic black, and other races. Education was divided into $<9$ th grade, 9-11th grade, high-school grade, college, and $\geq$ college graduate. The marital status was classified as follows: married, widowed, divorced, separated, never married, living with partner. The income level was based on the poverty income ratio, which was considered as a continuous variable in this study. Body mass index (BMI) was obtained from the examination document. Participants who smoke at least 100 cigarettes in their lifetime were considered as smokers. Alcohol use was defined as the consumption of at least 12 cups of alcoholic beverages in the last 12 months. The information regarding the presence of diabetes, hypertension, coronary heart disease, stroke, liver disease, and cancer were obtained from the questionnaire.

Detailed information about klotho, sleep-related variables and covariates is available at http://www.cdc.gov/ nchs/nhanes/.

\section{Statistical Analysis}

Statistical analysis was performed using $\mathrm{R}$ software (The R Foundation; https://www.r-project.org). The factors that influence the levels of klotho in the serum were detected using the univariate analysis. The association between sleep duration and serum klotho levels was assessed using multiple regression model. The threshold effect of sleep duration on serum klotho levels and the smoothing function were calculated using piecewise linear regression. The potential bias of the results due to the use of indicator variables with missing data was assessed by multiple imputation analysis. ${ }^{14}$

Two adjustment models were evaluated for the levels of klotho in the serum: the adjusted model I, which included variables in which the regression coefficients changed $>10 \%$ after the basic model was introduced or removed from the full model (age, race); the model II, 
which included variables in the model I and the regression coefficient of covariable to dependent variable of $P<0.1$ (age, race, gender, education level, marital status, smoking, alcohol use, hypertension, coronary heart disease, stroke, liver disease, cancer). ${ }^{15}$ A value of $P<0.05$ was considered to be statistically significant.

\section{Results}

The baseline characteristics of the study population are listed in Table 1. The mean age of the participants was $57.7 \pm 10.9$ years, and $51.6 \%$ were females. The mean sleep duration of the participants was $6.9 \pm 1.5$ hours, and $29.5 \%$ of them had trouble sleeping. The mean serum klotho concentration was $854.3 \pm 308.2 \mathrm{pg} / \mathrm{mL}$.

A univariate analysis of the potential influencing factors of the serum klotho level shown in Table 2 revealed that the concentration of klotho protein in the serum decreased when sleep duration increased $(P<0.001)$. In addition, trouble sleeping, age, gender, race, education level, marital status, smoking, alcohol use, hypertension, coronary heart disease, stroke, liver disease, and cancer were associated with the levels of klotho in the serum.

The correlation of the smooth curve fitting suggested a non-linear association between sleep duration and the level of klotho in the serum (Figure 1), and a two-piece linear regression model revealed an inflection point of 5.5 hours (Table 3). The multiple regression analysis shown in Table 4 after adjustment for model I and model II revealed a negative association between sleep duration and the concentration of klotho in the serum in the non-adjustment model $(\beta=-11.1$; 95\% CI: $-14.5,-7.6 ; P<0.001)$, adjustment model I $(\beta=$ $-7.3 ; 95 \% \mathrm{CI}:-10.8,-3.8 ; P<0.001)$ and adjustment model II $(\beta=-7.6 ; 95 \% \mathrm{CI}:-11.3,-4.0 ; P<0.001)$. The conversion of the sleep duration from a continuous variable to a categorical variable (tertile: T1: $<5.5$ hours; T2: 5.5-7.5 hours; T3: $>7.5$ hours) revealed that the level of klotho in the serum of the participants in the highest tertile ( $>7.5$ hours) was $21.9 \mathrm{pg} / \mathrm{mL}(95 \% \mathrm{CI}:-38.6,-5.2 ; P=0.010)$ lower than that in the lowest tertile ( $<5.5$ hours). No statistical difference on the concentration of klotho in the serum of the participants was observed between the middle tertile (5.5-7.5 hours) and the lowest tertile ( $<5.5$ hours) $(\beta=3.9$; 95\% CI: $-12.1,20.0$; $P=0.633$ ). Substitution analysis yielded consistent results, including multiple imputation of missing variables.

\section{Discussion}

To our knowledge, this work is the first reporting on the association between sleep duration and serum anti-aging
Table I Baseline Characteristics of the Study Participants

\begin{tabular}{|c|c|}
\hline Characteristics & Values \\
\hline Age(years), Mean \pm SD & $57.7 \pm 10.9$ \\
\hline \multicolumn{2}{|l|}{ Gender, N (\%) } \\
\hline Female & $7076(51.6)$ \\
\hline Male & $6649(48.4)$ \\
\hline \multicolumn{2}{|l|}{ Race, N (\%) } \\
\hline Mexican American & $2183(15.9)$ \\
\hline Other Hispanic & $1574(11.5)$ \\
\hline Non-Hispanic White & $5906(43.0)$ \\
\hline Non-Hispanic Black & $2712(19.8)$ \\
\hline Other Races & $1350(9.8)$ \\
\hline \multicolumn{2}{|l|}{ Education levels, $\mathrm{N}(\%)$} \\
\hline$\leq 9$ th Grade & $|87|(\mid 3.6)$ \\
\hline 9-1 Ith Grade & $2005(14.6)$ \\
\hline High School Grade & $304 \mid(22.2)$ \\
\hline College & $3701(27.0)$ \\
\hline$\geq$ College Graduate & $3099(22.6)$ \\
\hline \multicolumn{2}{|l|}{ Marital status, N (\%) } \\
\hline Married & $8196(59.7)$ \\
\hline Widowed & I I $48(8.4)$ \\
\hline Divorced & $2028(14.8)$ \\
\hline Separated & $542(4.0)$ \\
\hline Never married & $1147(8.4)$ \\
\hline Living with partner & $659(4.8)$ \\
\hline BMI $\left(\mathrm{kg} / \mathrm{m}^{2}\right)$, Mean $\pm \mathrm{SD}$ & $29.7 \pm 6.7$ \\
\hline \multicolumn{2}{|l|}{ Smoking, N (\%) } \\
\hline Yes & $6662(48.6)$ \\
\hline No & $7057(51.4)$ \\
\hline \multicolumn{2}{|l|}{ Alcohol use, N (\%) } \\
\hline Yes & $8996(70.6)$ \\
\hline No & $3738(29.4)$ \\
\hline \multicolumn{2}{|l|}{ Diabetes, N (\%) } \\
\hline Yes & $2462(18.0)$ \\
\hline No & 10,862 (79.2) \\
\hline Borderline & $391(2.9)$ \\
\hline \multicolumn{2}{|l|}{ Hypertension, N (\%) } \\
\hline Yes & $6375(46.5)$ \\
\hline No & $7335(53.5)$ \\
\hline \multicolumn{2}{|l|}{ CHD, N (\%) } \\
\hline Yes & $696(5.1)$ \\
\hline No & 12,974 (94.9) \\
\hline \multicolumn{2}{|l|}{ Stroke, N (\%) } \\
\hline Yes & $619(4.5)$ \\
\hline No & 13,086 (95.5) \\
\hline \multicolumn{2}{|l|}{ Liver Disease, N (\%) } \\
\hline Yes & $719(5.2)$ \\
\hline No & I2,977 (94.8) \\
\hline \multicolumn{2}{|l|}{ Cancer, N (\%) } \\
\hline Yes & $1593(11.6)$ \\
\hline No & 12,117 (88.4) \\
\hline \multicolumn{2}{|l|}{ Trouble sleeping, N (\%) } \\
\hline Yes & $4053(29.5)$ \\
\hline No & $9668(70.5)$ \\
\hline Sleep duration(hours), Mean \pm SD & $6.9 \pm 1.5$ \\
\hline Klotho $(\mathrm{pg} / \mathrm{mL})$, Mean $\pm \mathrm{SD}$ & $854.3 \pm 308.2$ \\
\hline
\end{tabular}

Abbreviations: SD, Standard deviation; N, Number; BMI, Body mass index; CHD, Coronary heart disease. 
Table 2 Univariate Analysis of Influencing Factors of the Serum Klotho Level

\begin{tabular}{|c|c|c|c|}
\hline \multirow[t]{2}{*}{ Variables } & \multirow[t]{2}{*}{ Values } & \multicolumn{2}{|c|}{ Klotho (pg/mL) } \\
\hline & & $\beta(95 \% \mathrm{Cl})$ & $P$ value \\
\hline Age(years), Mean \pm SD & $57.7 \pm 10.9$ & $-2.4(-2.9,-2.0)$ & $<0.001$ \\
\hline \multicolumn{4}{|l|}{ Gender, N (\%) } \\
\hline Female & 7076 (51.6\%) & Ref. & \\
\hline Male & $6649(48.4 \%)$ & $-45.4(-55.7,-35.1)$ & $<0.001$ \\
\hline \multicolumn{4}{|l|}{ Race, N (\%) } \\
\hline Mexican American & $2183(15.9 \%)$ & Ref. & \\
\hline Other Hispanic & 1574 (II.5\%) & $25.7(5.8,45.6)$ & 0.011 \\
\hline Non-Hispanic White & $5906(43.0 \%)$ & $-21.5(-36.6,-6.5)$ & 0.005 \\
\hline Non-Hispanic Black & $2712(19.8 \%)$ & $51.9(34.7,69.2)$ & $<0.001$ \\
\hline Other Races & $1350(9.8 \%)$ & $10.4(-10.4,31.3)$ & 0.327 \\
\hline \multicolumn{4}{|l|}{ Education level, $N(\%)$} \\
\hline$\leq 9$ th Grade & |87| (13.6\%) & Ref. & \\
\hline 9-IIth Grade & $2005(14.6 \%)$ & $2.9(-16.5,22.3)$ & 0.767 \\
\hline High School Grade & 304 I (22.2\%) & $-12.4(-30.2,5.3)$ & 0.170 \\
\hline College & 3701 (27.0\%) & $6.9(-10.3,24.0)$ & 0.433 \\
\hline$\geq$ College Graduate & $3099(22.6 \%)$ & $22.5(4.8,40.2)$ & 0.013 \\
\hline \multicolumn{4}{|l|}{ Marital status, N (\%) } \\
\hline Married & $8196(59.7 \%)$ & Ref. & \\
\hline Widowed & II 48 (8.4\%) & $-11.7(-30.7,7.4)$ & 0.23 \\
\hline Divorced & $2028(14.8 \%)$ & $10.7(-4.3,25.7)$ & 0.16 \\
\hline Separated & $542(4.0 \%)$ & $5.3(-21.5,32.1)$ & 0.699 \\
\hline Never married & II 47 (8.4\%) & $31.7(12.7,50.8)$ & 0.001 \\
\hline Living with partner & $659(4.8 \%)$ & $-6.8(-31.2,17.7)$ & 0.586 \\
\hline BMI $\left(\mathrm{kg} / \mathrm{m}^{2}\right)$, Mean \pm SD & $29.7 \pm 6.7$ & $-0.3(-1.1,0.4)$ & 0.394 \\
\hline \multicolumn{4}{|l|}{ Smoking, N (\%) } \\
\hline Yes & $6662(48.6 \%)$ & Ref. & \\
\hline No & 7057 (51.4\%) & $47.7(37.4,58.0)$ & $<0.001$ \\
\hline \multicolumn{4}{|l|}{ Alcohol use, N (\%) } \\
\hline Yes & $8996(70.6 \%)$ & Ref. & \\
\hline No & $3738(29.4 \%)$ & $55.6(43.9,67.3)$ & $<0.001$ \\
\hline \multicolumn{4}{|l|}{ Diabetes, N (\%) } \\
\hline Yes & $2462(18.0 \%)$ & Ref. & \\
\hline No & $10,862(79.2 \%)$ & $9.0(-4.4,22.5)$ & 0.189 \\
\hline Borderline & 391 (2.9\%) & $13.5(-19.4,46.4)$ & 0.421 \\
\hline \multicolumn{4}{|l|}{ Hypertension } \\
\hline Yes & $6375(46.5 \%)$ & Ref. & \\
\hline No & 7335 (53.5\%) & I8.5 (8.I, 28.8) & $<0.001$ \\
\hline \multicolumn{4}{|l|}{ CHD, N (\%) } \\
\hline Yes & $696(5.1 \%)$ & Ref. & \\
\hline No & 12,974 (94.9\%) & $5 I .4(27.9,74.9)$ & $<0.001$ \\
\hline \multicolumn{4}{|l|}{ Stroke, N (\%) } \\
\hline Yes & $619(4.5 \%)$ & Ref. & \\
\hline No & $13,086(95.5 \%)$ & $45.7(20.9,70.6)$ & $<0.001$ \\
\hline \multicolumn{4}{|l|}{ Liver Disease, N (\%) } \\
\hline Yes & 719 (5.2\%) & Ref. & \\
\hline No & I2,977 (94.8\%) & $-59.3(-82.4,-36.1)$ & $<0.001$ \\
\hline \multicolumn{4}{|l|}{ Cancer, N (\%) } \\
\hline Yes & 1593 (II.6\%) & Ref. & \\
\hline No & 12,117 (88.4\%) & $32.7(16.6,48.8)$ & $<0.001$ \\
\hline
\end{tabular}

(Continued) 
Table 2 (Continued).

\begin{tabular}{|l|l|l|l|}
\hline Variables & Values & \multicolumn{2}{|c|}{ Klotho (pg/mL) } \\
\cline { 3 - 4 } & & $\beta(95 \%$ Cl) & $P$ value \\
\hline Trouble sleeping, N (\%) & & & \\
Yes & $4053(29.5 \%)$ & Ref. & 0.036 \\
No & $9668(70.5 \%)$ & $12.1(0.8,23.4)$ & $<0.001$ \\
Sleep duration(hours), Mean \pm SD & $6.9 \pm 1.5$ & $-11.1(-14.5,-7.6)$ & \\
Sleep duration categories & & & \\
TI(<5.5 hours) & $2031(14.8 \%)$ & Ref. & 0.418 \\
T2(5.5-7.5 hours) & $6758(49.2 \%)$ & $-6.3(-21.6,9.0)$ & $<0.001$ \\
T3 $>7.5$ hours) & $4936(36.0 \%)$ & $-37.6(-53.5,-21.7)$ & \\
\hline
\end{tabular}

Abbreviations: SD, Standard deviation; N, Number; BMI, Body mass index; CHD, Coronary heart disease.

protein klotho concentration in the general population of the United States. A nonlinear association between sleep duration and serum klotho level was found. The levels of klotho protein in the serum of the participants whose sleep duration was more than 7.5 hours showed a downward trend as the duration of sleep increased.

Klotho protein is a one-way transmembrane protein, mainly including $\alpha$-klotho and $\beta$-klotho forms performing different functions. ${ }^{5,16} \alpha$-Klotho is a multifunctional protein regulating the metabolism of phosphate, calcium and vitamin $\mathrm{D},{ }^{5}$ while $\beta$-klotho is involved in key metabolic processes in various tissues. ${ }^{16}$ Although klotho gene expression is tissue specific, ${ }^{17,18}$ klotho gene defects

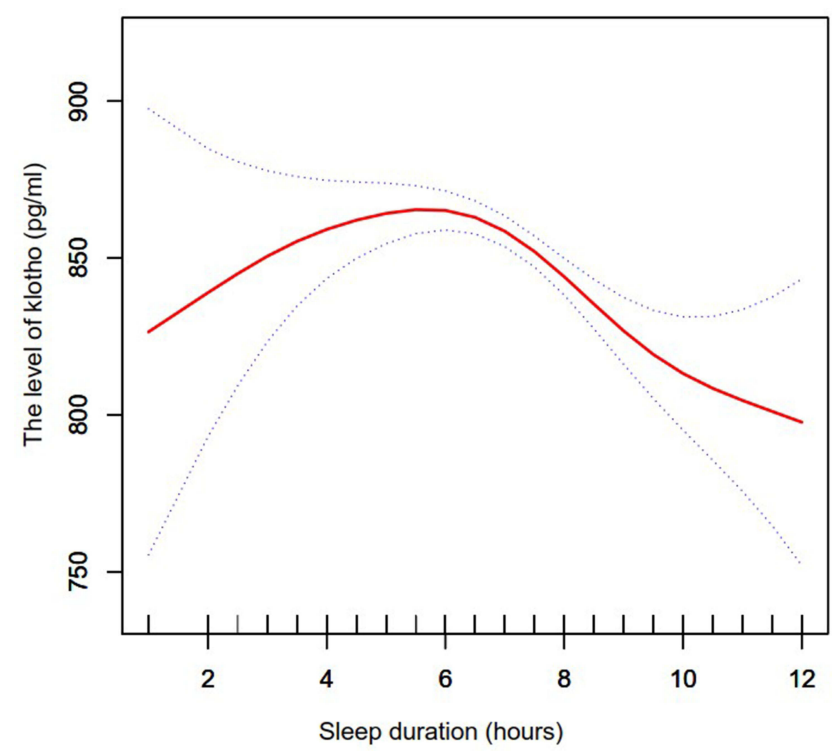

Figure I The fitted smooth curve showed the association between sleep duration and serum klotho levels after adjusting the relative confounding factors (age, race, gender, education level, marital status, smoking, alcohol use, hypertension, coronary heart disease, stroke, liver disease, cancer). The area between the dotted lines represents the $95 \%$ confidence interval. cause systemic phenotypes ${ }^{17}$ while klotho protein inhibits aging, ${ }^{19}$ suggesting that klotho protein may be involved in the regulation of the endocrine system. Mice lacking the klotho gene or fibroblast growth factor 23 show phosphate retention and premature aging syndrome, revealing that phosphate metabolism disorders may be the mechanism between klotho gene and aging. ${ }^{20}$ This evidence was used in this study as a basis to evaluate klotho protein in the serum as an aging-related marker.

Inappropriate sleep duration mainly determines the imbalance between the two sympathetic nervous systems and the hypothalamic-pituitary-adrenal axis. ${ }^{21}$ Moderate sleep duration is crucial for health. ${ }^{22}$ Insufficient sleep is a public health epidemic as revealed by the United States Centers for Disease Control (www.cdc.gov/features/ dssleep/). Insomnia and excessive sleep duration are both involved in the risk of inflammatory and infectious diseases, which in turn cause all-cause mortality. ${ }^{23-26} \mathrm{In}$ addition, sleep duration is also associated with some diseases related to aging, and an inverted U-shaped

Table 3 Threshold Effect Analysis of Sleep Duration on Serum Klotho Using the Two-Piecewise Regression Model

\begin{tabular}{|c|c|}
\hline Sleep Duration & Adjusted $\beta(95 \% \mathrm{Cl}), P$-value \\
\hline $\begin{array}{l}\text { Fitting by the standard linear } \\
\text { model } \\
\text { Fitting by the two-piecewise } \\
\text { linear model } \\
\text { Inflection point ( } 5.5 \text { hours) }\end{array}$ & $-7.62(-11.26,-3.98)<0.0001$ \\
\hline Sleep duration $<5.5$ hours & $12.85(-0.24,25.93) 0.0544$ \\
\hline Sleep duration $>5.5$ hours & $-12.64(-17.41,-7.87)<0.0001$ \\
\hline Log-likelihood ratio & 0.001 \\
\hline
\end{tabular}

Notes: Adjusted the covariates: age, race, gender, education level, marital status, smoking, alcohol use, hypertension, coronary heart disease, stroke, liver disease, cancer. 
Table 4 Multivariate Regression Analysis of the Association Sleep Duration (Hours) and Serum Klotho (pg/mL)

\begin{tabular}{|c|c|c|c|}
\hline & \multicolumn{3}{|c|}{ Serum Klotho $\beta(95 \% \mathrm{Cl}), P$-value } \\
\hline & Non-Adjusted & Adjust I & Adjust II \\
\hline & $-11.1(-14.5,-7.6)<0.001$ & $-7.3(-10.8,-3.8)<0.001$ & $-7.6(-11.3,-4.0)<0.001$ \\
\hline \multicolumn{4}{|l|}{$\begin{array}{l}\text { Sleep duration } \\
\text { Sleep duration categories }\end{array}$} \\
\hline $\mathrm{TI}(<5.5$ hours $)$ & Ref. & Ref. & Ref. \\
\hline T2(5.5-7.5 hours) & $-6.3(-21.6,9.0) 0.418$ & $3.2(-12.1,18.5) 0.679$ & $3.9(-12.1,20.0) 0.633$ \\
\hline $\mathrm{T} 3(>7.5$ hours $)$ & $-37.6(-53.5,-21.7)<0.001$ & $-22.0(-37.9,-6.0) 0.007$ & $-21.9(-38.6,-5.2) 0.010$ \\
\hline
\end{tabular}

Notes: Adjust I adjust for: age, race; Adjust Il adjust for: age, race, gender, education level, marital status, smoking, alcohol use, hypertension, coronary heart disease, stroke, liver disease, cancer.

association exists between sleep duration and cognitive aging. ${ }^{27}$ Insufficient sleep time is independently associated with an increased risk of atherosclerosis, ${ }^{1}$ and both insufficient and excessive sleep duration are related to an increased risk of cardiovascular disease. ${ }^{28,29}$ Elderly people with excessive sleep duration have a higher prevalence of stroke compared with elderly people with a sleep duration less than 9 hours. ${ }^{30}$ These studies revealed that inappropriate sleep duration is a very common and critical public health problem, but it is still overlooked, despite being easy to diagnose and treat. Our study demonstrated that excessive sleep duration ( $>7.5$ hours) was associated with a significant decrease in the anti-aging protein klotho, which is consistent with the previous evidence that excessive sleep duration causes aging.

In a randomized controlled study of 74 participants, Mochón-Benguigui et al report that sleep duration adjusted for fat mass and lean mass index was positively associated with soluble klotho levels. ${ }^{31}$ In comparison, the results of 13,765 American general population included in our work showed that serum klotho levels increased with sleep duration when sleep duration was within 5.5 hours after adjusting for confounding factors. This is consistent with the conclusion of Mochón-Benguigui et al. However, serum klotho levels were negatively correlated with sleep duration when sleep duration exceeded 7.5 hours. It revealed that excessive sleep duration may be detrimental to the level of serum anti-aging protein klotho. Although previous studies have already demonstrated that sleep duration is related to aging, no studies have reported the relationship between sleep duration and serum anti-aging protein klotho as we did in this work. Our research offers an additional evidence of sleep duration on aging by providing for the first time the relationship between sleep duration and the levels of klotho protein in the serum in the general population of the United States.
This study had several limitations. First of all, this analysis lacked participants' sleep details and sleep perception, which may influence the relationship between sleep duration and the concentration of klotho protein in the serum. Secondly, this study was a cross-sectional study. The causal association between sleep duration and serum klotho levels was not evaluated because of time constraints. Thus, the sleep duration was reported by the participants, with inevitable reported bias. Thirdly, this study may also be disturbed by other uncontrollable factors. For example, Pákó et al pointed out that lower plasma klotho levels were observed in patients with obstructive sleep apnea (OSA).$^{32}$ In addition, Oliveira et al found that the concentration of klotho decreased in the cerebrospinal fluid of narcolepsy patients. ${ }^{33}$ In our study population, whether the participants suffered from these diseases (eg, OSA, narcolepsy) and the proportion of these patients were unknown because such information was not available in the raw data. However, the evaluation was adjusted for several possibly important confounding factors (age; race; gender; education level; marital status; smoking; alcohol use; hypertension; coronary heart disease; stroke; liver disease; cancer). Moreover, this study used data from a large national survey in the United States (NHANES) from 2007 to 2016, which has a large sample size and random sampling, and a good representation of the general population in the United States.

\section{Conclusion}

Our study revealed that sleep duration was non-linearly related to the serum anti-aging protein klotho. Indeed, the level of the anti-aging protein klotho in the serum showed a significant downward trend when sleep duration exceeded 7.5 hours, thus being more at risk of agingrelated syndromes. Therefore, these people should monitor the level of the anti-aging protein klotho in the serum. 
Further well-designed prospective studies are needed to evaluate the effect of sleep duration on the anti-aging protein klotho to better understand the impact of the results obtained in this work on health, considering the enormous influence of sleep disorders on public health.

\section{Abbreviations}

NHANES, National Health and Nutrition Examination Survey; U, Uranium; OR, Odds ratio; CI, Confidence interval; GINA, Global Initiative for Asthma; US, United States; U, uranium; Pb, Lead; Hg, Mercury; As, Arsenic; BMI, Body mass index; ICP-MS, Inductively coupled plasma mass spectrometry; PIR, Poverty-to-income ratio; TP. Total protein; ALT, Alanine aminotransferase; AST, Aspartate aminotransferase; BUN, Blood urea nitrogen; Scr, Serum creatinine; TB, Total bilirubin; SUA, Serum uric acid; Ba, Barium; Cd, Cadmium; Co, Cobalt; Cs, Cesium; Mo, Molybdenum; Sb, Antimony; Tl, Thallium; Tu, Tungsten; CKD-EPI, Chronic Kidney Disease Epidemiology Collaboration; GFR, Glomerular filtration rate; OSA, Obstructive sleep apnea.

\section{Code Availability}

All analyses were performed using $\mathrm{R}$ (The R Foundation; https://www.r-project.org) software and Empower (X\&Y solutions, Inc., Boston, MA; http://www.empowerstats.com).

\section{Data Sharing Statement}

The data used in this study are publicly available on the Internet. https://www.cdc.gov/nchs/nhanes/.

\section{Ethics Approval and Consent to Participate}

Data analyzed in this study were from NHANES. Protocols involved were approved by the National Center for Health Statistics (NCHS) Research Ethics Review Board (ERB), and consent from all participants was documented. This study was a secondary analysis of the data, which was deemed exempt from review by the Ethics Committee of the Fourth Affiliated Hospital of Zhejiang University School of Medicine.

\section{Consent for Publication}

All authors consented for publication.

\section{Author Contributions}

All authors contributed to data analysis, drafting or revising the article, have agreed on the journal to which the article will be submitted, gave final approval for the version to be published, and agree to be accountable for all aspects of the work.

\section{Funding}

This study was supported by the Medical and Health Science and Technology Plan Project of Zhejiang Province (No. 2020KY627).

\section{Disclosure}

The authors declare that they have no competing interests.

\section{References}

1. Domínguez F, Fuster V, Fernández-Alvira JM, et al. Association of sleep duration and quality with subclinical atherosclerosis. $J$ Am Coll Cardiol. 2019;73:134-144. doi:10.1016/j.jacc.2018.10.060

2. Suh SW, Han JW, Lee JR, et al. Sleep and cognitive decline: a prospective nondemented elderly cohort study. Ann Neurol. 2018;83:472-482. doi:10.1002/ana.25166

3. Smiley A, King D, Bidulescu A. The association between sleep duration and metabolic syndrome: the NHANES 2013/2014. Nutrients. 2019;11:2582. doi:10.3390/nu11112582

4. Duffy JF, Zitting KM, Chinoy ED. Aging and circadian rhythms. Sleep Med Clin. 2015;10:423-434. doi:10.1016/j.jsmc.2015.08.002

5. Xu Y, Sun Z. Molecular basis of Klotho: from gene to function in aging. Endocr Rev. 2015;36:174-193. doi:10.1210/er.2013-1079

6. Klotho KM. Pflugers archives. Eur J Physiol. 2010;459:333-343. doi:10.1007/s00424-009-0722-7

7. Chen K, Wang S, Sun QW, et al. Klotho deficiency causes heart aging via impairing the Nrf2-GR pathway. Circ Res. 2021;128:492-507. doi:10.1161/circresaha.120.317348

8. Wang Y, Sun Z. Current understanding of klotho. Ageing Res Rev. 2009;8:43-51. doi:10.1016/j.arr.2008.10.002

9. Luo L, Hao Q, Dong B, Yang M. The Klotho gene G-395A polymorphism and metabolic syndrome in very elderly people. $B M C$ Geriatr. 2016;16:46. doi:10.1186/s12877-016-0221-6

10. Mencke R, Olauson H, Hillebrands JL. Effects of Klotho on fibrosis and cancer: a renal focus on mechanisms and therapeutic strategies. Adv Drug Deliv Rev. 2017;121:85-100. doi:10.1016/j.addr.2 017.07.009

11. Wang $\mathrm{HL}, \mathrm{Xu} \mathrm{Q}$, Wang $\mathrm{Z}$, et al. A potential regulatory single nucleotide polymorphism in the promoter of the Klotho gene may be associated with essential hypertension in the Chinese Han population. Clin Chim Acta. 2010;411:386-390. doi:10.1016/j. cca.2009.12.004

12. Dong X, Milholland B, Vijg J. Evidence for a limit to human lifespan. Nature. 2016;538:257-259. doi:10.1038/nature19793

13. Khan SS, Singer BD, Vaughan DE. Molecular and physiological manifestations and measurement of aging in humans. Aging Cell. 2017;16:624-633. doi:10.1111/acel.12601

14. Melamed A, Margul DJ, Chen L, et al. Survival after minimally invasive radical hysterectomy for early-stage cervical cancer. N Engl J Med. 2018;379:1905-1914. doi:10.1056/NEJMoa1804923

15. Wang S, Zhang J, Lu X. Non-linear association of plasma level of high-density lipoprotein cholesterol with endobronchial biopsy bleeding in patients with lung cancer. Lipids Health Dis. 2019;18:17. doi:10.1186/s12944-019-0966-y

16. Lee S, Choi J, Mohanty J, et al. Structures of $\beta$-klotho reveal a 'zip code'-like mechanism for endocrine FGF signalling. Nature. 2018;553:501-505. doi:10.1038/nature25010 
17. Kuro-o M, Matsumura Y, Aizawa H, et al. Mutation of the mouse klotho gene leads to a syndrome resembling ageing. Nature. 1997;390:45-51. doi:10.1038/36285

18. Ben-Dov IZ, Galitzer H, Lavi-Moshayoff V, et al. The parathyroid is a target organ for FGF23 in rats. J Clin Invest. 2007;117:4003-4008. doi:10.1172/jci32409

19. Kurosu H, Yamamoto M, Clark JD, et al. Suppression of aging in mice by the hormone Klotho. Science. 2005;309:1829-1833. doi:10.1126/science. 1112766

20. Razzaque MS, Sitara D, Taguchi T, St-Arnaud R, Lanske B. Premature aging-like phenotype in fibroblast growth factor 23 null mice is a vitamin D-mediated process. FASEB J. 2006;20:720-722. doi:10.1096/fj.05-5432fje

21. Irwin MR. Why sleep is important for health: a psychoneuroimmunology perspective. Ann Rev Psychol. 2015;66:143-172. doi:10.1146/annurev-psych-010213-115205

22. Buysse DJ. Sleep health: can we define it? Does it matter? Sleep. 2014;37:9-17. doi:10.5665/sleep.3298

23. Dew MA, Hoch CC, Buysse DJ, et al. Healthy older adults' sleep predicts all-cause mortality at 4 to 19 years of follow-up. Psychosom Med. 2003;65:63-73. doi:10.1097/01.psy.0000039756.23250.7c

24. Kripke DF, Garfinkel L, Wingard DL, Klauber MR, Marler MR. Mortality associated with sleep duration and insomnia. Arch Gen Psychiatry. 2002;59:131-136. doi:10.1001/archpsyc.59.2.131

25. Mallon L, Broman JE, Hetta J. Sleep complaints predict coronary artery disease mortality in males: a 12-year follow-up study of a middle-aged Swedish population. J Intern Med. 2002;251:207-216. doi:10.1046/j.1365-2796.2002.00941.x
26. Vgontzas AN, Fernandez-Mendoza J, Liao D, Bixler EO. Insomnia with objective short sleep duration: the most biologically severe phenotype of the disorder. Sleep Med Rev. 2013;17:241-254. doi:10.1016/j.smrv.2012.09.005

27. Leng Y, Yaffe K. Sleep duration and cognitive aging-beyond a U-shaped association. JAMA Netw Open. 2020;3:e2014008. doi:10.1001/jamanetworkopen.2020.14008

28. Tobaldini E, Fiorelli EM, Solbiati M, et al. Short sleep duration and cardiometabolic risk: from pathophysiology to clinical evidence. Nat Rev Cardiol. 2019;16:213-224. doi:10.1038/s41569-018-0109-6

29. Cappuccio FP, Cooper D, D’Elia L, Strazzullo P, Miller MA. Sleep duration predicts cardiovascular outcomes: a systematic review and meta-analysis of prospective studies. Eur Heart J. 2011;32:1484-1492. doi:10.1093/eurheartj/ehr007

30. Fang J, Wheaton AG, Ayala C. Sleep duration and history of stroke among adults from the USA. J Sleep Res. 2014;23:531-537. doi:10.1111/jsr. 12160

31. Mochón-Benguigui S, Carneiro-Barrera A, Castillo MJ, AmaroGahete FJ. Is sleep associated with the S-Klotho anti-aging protein in sedentary middle-aged adults? The FIT-AGEING study. Antioxidants. 2020;9. doi:10.3390/antiox9080738

32. Pákó J, Kunos L, Mészáros M, et al. Decreased levels of anti-aging Klotho in obstructive sleep apnea. Rejuvenation Res. 2020;23 (3):256-261. doi:10.1089/rej.2019.2183

33. da Paz Oliveira G, Elias RM, Peres Fernandes GB, et al. Decreased concentration of klotho and increased concentration of FGF23 in the cerebrospinal fluid of patients with narcolepsy. Sleep Med. 2021;78:57-62. doi:10.1016/j.sleep.2020.11.037
International Journal of General Medicine

\section{Publish your work in this journal}

The International Journal of General Medicine is an international, peer-reviewed open-access journal that focuses on general and internal medicine, pathogenesis, epidemiology, diagnosis, monitoring and treatment protocols. The journal is characterized by the rapid reporting of reviews, original research and clinical studies across all disease areas. The manuscript management system is completely online and includes a very quick and fair peer-review system, which is all easy to use. Visit http://www.dovepress.com/ testimonials.php to read real quotes from published authors. 